ช

OPEN ACCESS

ÉDITEURS

- Miguel Oliveira, Jr. (UFAL)

- René Almeida (UFS)

ÉVALUATEURS

- Ida Machado (UFMG)

- Débora Massmann (UFAL)

DATES

- Reçu: 10/11/2020

- Acceptée: 02/12/2020

- Publié: 14/02/2021

\section{COMMENT CITER}

MAINGUENEAU, Dominique (2021). Aux limites de l'analyse du discours.

Cadernos de Linguística, v. 2, n. 1, p. 01 15.

\title{
AUX LIMITES DE L'ANALYSE DU DISCOURS
}

\author{
Dominique MAINGUENEAU (D) $\boldsymbol{\Delta}$ \\ Sorbonne Université (Paris)
}

\section{RÉSUMÉ}

Cette contribution s'attache à mettre en évidence certaines limites de l'analyse du discours actuelle. Celle-ci est censée étudier les manifestations du discours dans toute leur diversité, mais la plupart des analystes du discours n'étudient qu'un ensemble restreint de données : d'une part les conversations, d'autre part certains secteurs de la société : la politique, les médias, l'éducation, l'économie, la santé, la justice. Ils tiennent en effet pour évidente une certaine conception du discours qui est centrée sur l'interaction conversationnelle et le couple texte/genre et qui rejette un masse considérable de données à la périphérie. Ils voient le discours comme une interaction entre des individus qui agissent dans des contextes bien définis et qui développent des stratégies en fonction de leurs intérêts pour s'influencer l'un l'autre et modifier la situation. On peut soutenir au contraire que les pratiques discursives reposent sur divers régimes de la communication et doivent, en conséquence, être analysés avec des concepts et des méthodes spécifiques. J'évoque divers phénomènes qui mettent en cause le modèle dominant : les discours constituants, Internet, les locuteurs non-humains, la communication avec les animaux, l'auctorialité, les "énoncés adhérents".

\section{ABSTRACT}

This contribution aims to outline some limits of discourse analysis today. Discourse analysis is not supposed to privilege any specific kind of data, but, most discourse analysts focus on a restricted area within the manifold manifestations of discourse : conversations, politics, the media, education, business, the health system, justice. They take for granted that 
discourse must be modeled after conversational practices, and/or that the basic relevant unit of discourse analysis is the pair text/genre. They see discourse as an interaction between flesh-and-blood people who act in well defined settings and develop strategies in keeping with their own aims and interests in order to influence each other and modify the situation. On the contrary, it can be argued that such a standard is not relevant for a wide range of data, that discourse practices can be divided into various regimes of communication and must accordingly be analyzed with specific concepts and toolkits. In this article, I discuss various phenomena that call this standard into question: self-constituting discourses, the Internet, non-human speakers, communication with animals, authorship, "adhering utterances".

\section{MOTS-CLÉS}

Analyse du Discours ; Discours Constituants ; Web ; Locuteurs NonHumains ; Énoncés Adhérents.

\section{KEYWORDS}

Discourse Analysis; Self-Constituting Discourses; Web; Non-Human Speakers; Adhering Utterances. 


\section{INTRODUCTION}

Ceux qui travaillent dans le vaste champ des études de discours peuvent avoir des attitudes très diverses à l'égard de leur objet. Déjà, on peut distinguer entre ceux qui travaillent dans le cadre de la "théorie du discours" et les "analystes du discours" proprement dits, qui étudient des corpus en s'appuyant en général sur les sciences du langage (ANGERMULLER, MAINGUENEAU, WODAK (eds), 2014, p. 5-7). Parmi ces derniers, certains utilisent l'analyse du discours comme une boite à outils, un ensemble de concepts et de méthodes qui leur permettent, par l'étude de corpus appropriés, à répondre à des questionnements définis par d'autres disciplines. D'autres, animés par une visée résolument critique, ont une conception qu'on pourrait dire "thérapeutique" : ils cherchent à remédier à des dysfonctionnements sociaux que rend possibles le discours. D'autres enfin cherchent à éclairer le fonctionnement du discours, et pas seulement de répondre à des demandes issues d'autres disciplines. On pourrait penser que ces derniers s'interrogent sur l'extension de leur champ d'étude : quelles données relèvent de ce "discours" qu'ils entendent étudier? En réalité, cette question est rarement posée : soit parce que les chercheurs préfèrent réfléchir sur la discursivité en général et ne se préoccupent pas de la diversité empirique de ses manifestations, soit parce qu'ils travaillent sur un ensemble limité de données (les médias, la politique, l'éducation, etc.) et n'éprouvent pas le besoin d'envisager l'ensemble des manifestations du discours. Mais dans l'esprit de la plupart des analystes du discours cette restriction de leur champ de vision n'est pas un problème : certes, ils n'étudient eux-mêmes que certains types de données, mais il existe d'autres chercheurs qui abordent celles qu'ils ne prennent pas en compte.

Malheureusement, quand on regarde quels types de données sont effectivement abordées, il apparaît que les analystes du discours ne s'intéressent pas à toutes les manifestations du discours, loin de là. Ils étudient les conversations "ordinaires" et un certain nombre de secteurs de la production discursive - essentiellement les médias, la politique, l'éducation, le business, la justice, la santé - qui sont d'ailleurs des objets privilégiés dans les sciences sociales. Certes, c'est là un champ de recherche considérable, mais d'autres types de données qui jouent un rôle important dans la société ne sont que marginalement prises en compte.

Cette marginalisation s'appuie implicitement sur une certaine conception de la discursivité, ou plutôt sur deux conceptions de la discursivité qui partagent certains présupposés. Selon la première, le discours ne s'exerce pleinement que dans l'interaction orale ; selon la seconde, l'unité de base du discours est le texte, oral ou écrit, associé à un cadre générique. On retrouve là les deux grands courants qui structurent le champ des études de discours : la tradition nord-américaine, qui privilégie l'oralité conversationnelle, et la tradition 
européenne qui se focalise sur les institutions!. Mais, quelles que soient leurs divergences, ces deux traditions partagent tacitement une certaine conception du discours, appréhendé comme une interaction réelle ou virtuelle entre des locuteurs inscrits dans des cadres sociaux bien définis et qui cherchent à s'influencer en développant des stratégies discursives en fonction de leurs intérêts.

II n'est pas question de contester la pertinence de cette conception de la discursivité à laquelle se rattache d'ailleurs une bonne part de mes propres travaux -, mais il est permis de se demander si elle ne limite pas a priori et indument le champ du discours. Si les analystes du discours entendent être en prise sur la société, sur la complexité des pratiques effectives des locuteurs, ils peuvent difficilement ne pas se poser des questions à ce propos. On ne peut par exemple difficilement ignorer l'importance du fait religieux dans le monde contemporain, ne serait-ce que sur le plan géopolitique ; mais bien rares sont les travaux d'analyse du discours qui portent sur ce sujet (MAINGUENEAU 2009, 2017) et, quand ils le font, c'est bien souvent pour répondre à des urgences sociales (le terrorisme par exemple) et non en s'intéressant au discours religieux. On ne peut pas non plus ignorer que les humains passent de plus en plus de temps à consommer des énoncés d'ordre esthétique. Certes, les séries de Netflix ou d'Amazon ne relèvent pas du même univers que les oeuvres de Pessoa ou de Machado de Assis, mais comment les ignorer? Et que dire des jeux vidéo, qui brouillent la frontière traditionnelle entre jeu et littérature ?

\section{LES DISCOURS CONSTITUANTS}

Du seul fait que je m'intéresse depuis de nombreuses années à ce que j'appelle les « discours constituants " (MAINGUENEAU, 1999), j'ai pu constater le peu d'attention que prêtent la plupart des analystes du discours à ce qui relève du religieux ou de l'esthétique. Ce désintérêt, qui porte plus largement sur l'ensemble des discours constituants, peut s'expliquer par la convergence de multiples facteurs, mais en général il ne fait l'objet d'aucune justification théorique. Comme dans les phénomènes de discrimination sociale, l'exclusion est d'autant plus efficace et puissante qu'elle repose sur une évidence partagée, qu'elle n'est pas explicitée. L'analyse du discours s'est ainsi maintenue à distance des départements universitaires qui traditionnellement étudient les écrits

1 Ces "traditions" ne sont pas des domaines étanches, en tout cas pas sur le plan géographique. On trouve aujourd'hui des représentants des deux sur tous les continents. La problématique du genre de discours, par exemple, n'est pas bien différente dans l'analyse du discours francophone (CHARAUDEAU, 1995 ; MAINGUENEAU, 1998 ; RASTIER, 2001) britannique (SWALES, 1990 ; FAIRCLOUGH, 2003) et nord-américaine (BAZERMANN, 1988). Quant à l'analyse conversationnelle, une part de ses recherches est consacrée aux interactions dans les institutions (HERITAGE, 2005; HERITAGE \& CLAYMAN, 2010). 
prestigieux (littérature, philosophie, religion...), renvoyant leur étude à des spécialistes, qui eux-mêmes sont trop contents de conserver le monopole en la matière. II est indéniable que l'étude du discours politique, de la publicité ou des émissions de télévision permet de mettre facilement en relation des phénomènes linguistiques et des facteurs sociaux, alors que ce lien est beaucoup plus complexe à établir lorsqu'on analyse des corpus extraits des discours constituants. Mais on ne peut pas dire que ce soit là une justification d'ordre épistémologique.

Les corpus extraits des discours constituants ne sont cependant pas totalement ignorés des analystes du discours, mais quand ils les abordent c'est à travers des données qui leur sont plus familières : une émission littéraire à la télévision et non Madame Bovary, un sermon et non un évangile ou une épitre de saint Paul, des magazines de vulgarisation scientifique et non des articles de physique ou de médecine... II est vrai que les textes de référence des discours constituants posent problème à un analyste du discours : outre le fait que leur contenu est d'un accès souvent difficile, ils mobilisent des dispositifs d'énonciation inhabituels. Par exemple Dieu ou Platon, chacun à sa façon, ne sont pas des locuteurs ordinaires, les textes qu'on leur attribue ont une matérialité spécifique et ne sont lisibles qu'à travers des institutions et des traditions.

Considérons par exemple la notion de "parole de Dieu". Les religions du Livre (le judaïsme, le christianisme et l'Islam) partent du postulat que Dieu nous parle ; sans être un humain qui produit des énoncés, il s'adresse aux hommes, à travers des textes sacrés qui délivrent son message. Chaque croyant est ainsi appelé à comprendre, à travers ces textes, les intentions de Dieu à son égard. Il est clair qu'une telle "parole" ne rentre pas dans le cadre de la communication verbale telle qu'on l'appréhende communément dans les sciences du langage. Non seulement l'initiateur du message, Dieu, n'est pas un locuteur mais il ne partage pas le même monde que ses destinataires: parce qu'il est censé appartenir au monde surnaturel, mais aussi parce que les textes sacrés qui recèlent son message ont été rédigés dans un contexte historique bien différent de celui des fidèles. Cette relation de communication très singulière implique donc l'intervention de médiateurs : d'une part ceux qui ont rédigé les textes, mus par une inspiration divine, d'autre part ceux qui ont pour fonction de les rendre pertinents pour des destinataires qui varient selon les lieux et selon les époques. Ce qui requiert chez ces interprètes non seulement la maîtrise de procédures d'interprétation mais aussi une relation privilégiée avec la divinité : si on n'est pas d'une certaine façon habité par l'Esprit, l'énoncé ne fera pas véritablement sens, Dieu ne parlera pas à travers le texte.

Il n'y a donc pas énonciation directe de Dieu, mais une interprétation qui est convertie en énonciation indirecte : "dans ce texte Dieu nous enseigne que... ». Un tel dispositif implique un " cadre herméneutique " (MAINGUENEAU, 2007), le postulat que le sens véritable de l'énoncé n'est pas immédiatement accessible, qu'il faut une exégèse pour le mettre au jour: même les textes qui paraissent les plus transparents exigent du destinataire qu'il 
dérive des significations cachées. Aucun défaut dans l'énoncé ou dans l'énonciation n'est possible de la part de Dieu ; s'il y a des problèmes de compréhension, ils ne peuvent venir que de l'homme. Le texte a beau sembler obscur ou manquer de pertinence, le cadre herméneutique garantit qu'à un niveau supérieur la faute n'est qu'apparente.

On le voit, pour appréhender à sa juste mesure des énonciations de ce type il faut prendre ses distances avec certains présupposés du modèle de la communication qui prévaut en analyse du discours.

Au-delà des problèmes d'analyse empirique que pose le fonctionnement des discours constituants, leur existence même amène à s'interroger sur l'homogénéité de l'espace où se manifeste le discours. Une des caractéristiques fondamentales des discours constituants est qu'ils tirent leur autorité d'être " paratopiques " : leur appartenance à la société est radicalement problématique et ils se nourrissent de cette intenable position. Comme ils ne s'inscrivent pas dans l'espace du discours de la même manière que les autres discours, on est en droit de penser qu'il faut adapter en conséquence notre appareil conceptuel et méthodologique. On peut se poser des questions du même ordre à propos des discours "atopiques ", comme la pornographie : le refoulement auxquels ils sont soumis n'est pas un accident, il est constitutif. Loin d'occuper de plein droit l'espace du discours, ils circulent dans les interstices des paroles autorisées, tout à la fois visibles et invisibles. Peut-on les étudier comme des corpus de presse ou de publicité ?

Qu'il s'agisse de paratopie ou d'atopie, l'univers du discours n'apparaît pas monotone, il ne se présente pas comme une immense carte de géographie où les zones d'activité discursive seraient simplement juxtaposées, mais comme un lieu de tension entre des régimes de discours qui ne s'inscrivent pas de la même manière dans l'espace.

\section{WEB ET "LOCUTEURS ANGELIQUES"}

Paratopie et atopie relèvent du régime traditionnel du discours ; il n'en va pas de même pour les énoncés que rendent possibles les nouvelles technologies de communication - en particulier Internet -, qui bouleversent la représentation que l'on peut se faire de l'univers du discours. Aujourd'hui, n'importe qui, à partir du moment où il dispose d'une connexion, produit et consomme des énoncés sur deux espaces, dont l'un ("virtuel ») résulte de l'interaction d'une multitude de machines. L'analyse du discours, façonnée par le monde dans lequel elle est apparue, les années 1960, peine à adapter son appareil conceptuel et méthodologique à cette nouvelle donne, où des catégories que l'on pensait relativement stables se trouvent brutalement mises en cause : texte, locuteur, destinataire, genre de discours, écriture, énonciation, mémoire, etc. Il est devenu impossible, comme on l'a fait longtemps, de considérer la technologie comme un simple élément du "contexte": c'est un véritable acteur du processus de 
communication. Une telle transformation concerne les données - puisque se développent de nouveaux types de productions sémiotiques - mais aussi les conditions mêmes de la recherche, qui dépendent de plus en plus de logiciels et de bases de données.

Pourtant, la plupart des analystes du discours semblent vivre dans un monde où le faceà-face conversationnel et l'imprimé sont encore la norme de la communication. Si l'on considère les manuels et les articles qui se réclament de l'analyse du discours, un rôle périphérique est donné aux corpus produits par les nouvelles technologies, sauf si on peut les étudier en utilisant les concepts usuels en analyse de conversations : chats, forums, courrier électronique... La plupart du temps le web sert surtout à fournir des corpus qui sont étudiés comme s'il s'agissait d'énoncés verbaux ordinaires. Ce qui laisse en suspens une question essentielle : les énoncés produits avec les nouvelles technologies relèvent-ils du même régime de discours que l'oral et l'écrit? II est permis d'en douter.

J'ai eu par exemple l'occasion de me rendre compte (MAINGUENEAU, 2013) qu'une notion aussi cruciale pour l'analyse du discours que le genre de discours n'est guère opératoire quand on aborde le Web. Bien sûr, les sites Web peuvent être divisés en grandes catégories (blogs, réseaux sociaux, sites d'information...), mais ces catégories ne sont pas des genres au sens qu'a habituellement ce terme en analyse du discours. Alors que la conception habituelle du genre implique une carte des activités verbales (on rapporte les genres de discours à divers types de discours qui correspondent à des secteurs bien différenciés de l'activité sociale), le Web implique une "dé-différenciation" des domaines du discours.

A l'instar de celle de genre, la notion de "page" se révèle tout aussi problématique. Une "page" sur le web ne peut être appréhendée d'un seul coup d'œil : l'écran n'affiche qu'une partie d'un ensemble qui ne peut être considéré comme une unité. Sur de nombreux sites on perçoit des mosaïques de modules hétérogènes : publicités, slogans, vidéos, citations, débuts d'articles, schémas, photos... Beaucoup de ces modules ne sont pas autonomes : ce ne sont que des fragments qui, lorsqu'on clique dessus, donnent accès à d'autres pages du même site ou d'autres sites et les textes eux-mêmes ne sont pas des entités closes : ils contiennent des liens qui ouvrent sur d'autres pages ou d'autres sites. Le Web apparaît de plus en plus comme un monde de morceaux décontextualisés qui peuvent être combinés d'innombrables façons, indépendamment d'une référence à une totalité de texte dont ils feraient partie. Même l'identité d'une page devient problématique; ce qui peut être vu à l'écran n'existe qu'à un moment précis : certains modules restent stables pendant une certaine période, d'autres sont mis à jour de temps en temps, d'autres enfin changent sans cesse (le score d'un match de tennis, les cours de la Bourse...).

De manière plus générale, ces innovations techniques mettent en question le présupposé selon lequel la communication va d'une instance douée de conscience à une autre, ou encore celui selon lequel les énoncés peuvent être véritablement situés, référés à une source identifiable et à un lieu. On peut même aller plus loin : notre existence quotidienne 
nous fait de plus en plus entrer en contact avec des messages énoncés par des êtres non humains - que j'ai proposé d'appeler des "locuteurs angéliques" (MAINGUENEAU, 2014 , p. 195) - qui s'adressent à nous oralement mais ne sont pas, à proprement parler, des locuteurs : messages sur les répondeurs automatiques, consignes données par les appareils G.P.S. ou les caisses automatiques de paiement dans les supermarchés, assistants sur les ordinateurs ou les smartphones, etc. II ne s'agit pas seulement d'énonciations monologiques, de la machine vers l'homme. Tout le monde a fait l'expérience de ces difficiles " dialogues " que l'on peut avoir avec les voix qui dans de nombreuses administrations ou entreprises sont chargées de répondre aux appels téléphoniques et guider les usagers vers le bon interlocuteur. On est ici très loin de la parole de Dieu, mais dans un cas comme dans l'autre on a affaire à une communication foncièrement asymétrique entre des non-humains et des humains qui implique des médiations complexes : institutionnelles et textuelles pour Dieu, technologiques pour les machines. À ces énonciations orales il faudrait ajouter les textes écrits qui sont générés par des logiciels : résumés, prévisions météorologiques, rapports techniques, etc. Certains programmes écrivent même des poèmes ou des romans. Sans aller si loin, l'écran d'un smartphone ou d'un ordinateur ne cesse d'afficher des messages à destination des usagers.

\section{HUMAINS ET ANIMAUX}

Il existe dans un tout autre domaine des "dialogues " foncièrement asymétrique d'un type bien différent : entre humains et animaux domestiques. II existe de plus en plus de travaux sur cette question, dans la perspective plus large d'un intérêt pour le « non-humain » issu en grande partie de l'anthropologie de la nature (DESCOLA, 2005) ou des théories de l'acteurréseau (AKRICH; CALLON; LATOUR, 2006 ; HOUDART; THIERY (eds), 2011). Les études sur les relations entre humains et animaux se sont institutionnalisées (ARLUKE; SANDERS, 1996 ; SANDERS, 2003 ; GOODE, 2007 ; GUILLO, 2009)) et ont gagné les études de discours (MONDÉMÉ, 2018, 2019). Dans un pays comme la France on évalue à environ 8 millions le nombre de chiens et plus de 14 millions le nombre de chats : autant d'êtres avec lesquels les humains passent un temps considérable à interagir verbalement. Et si l'on y ajoute les chevaux et les bovins, cela fait une population considérable. II existe même dans les cultures agraires des pratiques discursives ritualisées d'adresse aux animaux ; en France ces pratiques appartiennent au passé : le "briolage" par exemple consistait à soutenir par la parole le travail des bœufs. La voix, au plus près le rythme des bêtes, accompagnant l'effort de ses sons, de ses mots, et parfois de phrases longues ou de textes, des sortes de poèmes que chaque région ou chaque laboureur déclinait à sa manière (PATRIMOINE CULTUREL IMMATÉRIEL, 2012). Le problème est de savoir à quel titre on peut exclure de l'univers du discours cette 
communication entre espèces. Si on l'intègre au champ du discours, on est bien obligé de prendre en compte son irréductible spécificité : pour énoncer, l'humain qui interagit avec l'animal est contraint de se décaler de son humanité, mais aussi de décaler son "interlocuteur" de son animalité.

\section{L'AUTEUR}

II n'est même pas besoin d'évoquer les discours constituants, les nouvelles technologies ou les animaux domestiques pour faire apparaître des sources d'énonciation qui échappent au modèle usuel de la communication. II suffit tout simplement de songer à la notion d'auteur que, significativement, la plupart des analystes du discours évitent, sans doute parce qu'elle ne relève que de l'écrit. Ils préfèrent parler de « locuteur » et abandonner cette notion aux théoriciens de la littérature.

En réalité, le terme "auteur" peut être employé de deux manières très différentes. Dans son premier emploi il concerne n'importe quel texte écrit, et devrait donc intéresser les analystes du discours ; il désigne un être qui n'est pas I'"énonciateur", c'est-à-dire une catégorie linguistique, ni un être humain appréhendé hors de la langue, mais une fonction qui combine l'agentivité ( $X$ réalise une énonciation) et une dimension juridique ( $X$ doit en répondre). La second emploi, en revanche, est difficilement compatible avec les présupposés dominants en analyse du discours; dans l'acception précédente on a affaire à des instances associées à des textes relevant de genres ("I'auteur de cette lettre, de ce rapport..."), alors que dans celle-ci l'auteur est le corrélat d'une "OEuvre » majuscule constituée d'un nombre plus ou moins grand de textes relevant de genres divers mais censés exprimer la vision du monde d'une personnalité singulière. L'“ OEuvre » est une catégorie que les analystes du discours ignorent généralement, alors même que dans une société (l'analyse du discours elle-même ne cesse d'évoquer les textes de Bakhtine, Foucault, Goffman...), on s'appuie constamment sur un ensemble restreint de textes référés à de "grands auteurs ". C'est sur cette notion que réfléchit M. Foucault dans un article célèbre: "Qu'est-ce qu'un auteur ?" (1969). Pour clarifier la terminologie, j'appelle « auctor » cet être à la source d'une OEuvre.

Il faut l'intervention de tiers pour qu'un locuteur ou une locutrice devienne ainsi auctor. En tant que fragment d'une OEuvre, un texte n'est plus lu comme le produit d'une activité discursive située mais comme l'expression d'un Sujet extra-ordinaire. On sort de la logique de l'échange verbal ordinaire, celle du texte et du genre. On n'a plus en effet affaire à un "locuteur " ou une "locutrice ", quelqu'un qui produit un énoncé relevant d'un genre de discours à l'attention de destinataires qui partagent son monde. Ceux à qui s'adressent les textes appartenant aux "OEuvres" ne sont pas spécifiés par les genres correspondants, mais constituent un public indéterminé. Lorsque Clarice Lispector écrivait sa 
correspondance, publiait des romans ou écrivait des articles dans les journaux, elle s'inscrivait dans des routines discursives disponibles dans son environnement et s'adressait à des destinataires spécifiés par ces routines ; lorsque nous lisons ces mêmes textes dans ses "OEuvres complètes ", ils s'adressent à un public indéterminé et sont lus comme des fragments d'une OEuvre qui exprime un point de vue unique sur le monde que ses commentateurs s'attachent à expliciter.

\section{LES ENONCES ADHERENTS}

Aux antipodes des OEuvres, qui portent à leur paroxysme les formes de textualité les plus classiques, il existe une autre masse considérable de données que négligent presque totalement les analystes du discours : ce que j'appelle les "énoncés adhérents" (MAINGUENEAU, 2020, p. 115). On les trouve partout dans notre environnement : " I love New York » sur un mug, un panneau "Légumes " dans un supermarché, une étiquette sur une boîte de conserve ou une bouteille de jus de fruit, une phrase sur un T-shirt, une enseigne de magasin (" boulangerie ", " pharmacie »...), un tatouage, des consignes de lavage cousues sur un vêtement, une épitaphe sur une tombe, une plaque indiquant le nom d'une rue, le nom de l'entreprise sur l'uniforme d'un employé... Ces énoncés figurent aujourd'hui sur une foule de supports qui en étaient dépourvus auparavant (par exemple les animaux d'élevage ou les animaux domestiques) ou sont de plus en plus complexes (ainsi sur les emballages des produits alimentaires).

L'énoncé adhérent et son support ne forment pas un tout compact, comme le feraient deux pièces complémentaires d'un mécanisme : le vin n'a pas besoin de l'étiquette sur la bouteille pour être bu, ni la rue de la plaque où est indiqué son nom pour être une rue. Mais en ajoutant des énoncés adhérents à des objets qui en étaient jusque là dépourvus ou en modifiant des énoncés adhérents déjà existants, nous conférons une nouvelle identité à ces objets, nous les inscrivons dans de nouveaux réseaux de relations et de nouvelles pratiques. Une réflexion sur les énoncés adhérents met ainsi en cause l'idée spontanée qu'il y aurait deux mondes bien disjoints : celui des choses et celui des mots. Les objets au milieu desquels nous vivons ne cessent de se transformer en fonction des énoncés qui y adhèrent.

Le désintérêt de l'analyse du discours pour ces énoncés adhérents peut s'expliquer ici encore par les présupposés attachés au modèle dominant de l'activité discursive, qui voit dans toute énonciation une interaction communicationnelle explicite ou implicite. A propos des tatouages, par exemple, M.-A. Paveau fait remarquer que " dire, ce n'est pas forcément dire à quelqu'un, ce peut être dire tout court, dire pour soi ou simplement exprimer, marquer ou formuler son expérience, installer un lien avec le réel » $(2009,2.3$.$) . B.$ 
Bosredon (1997, p. 18) souligne aussi ce point quand il étudie les étiquettes placées à côté des tableaux dans les musées:

\footnotetext{
Une étiquette n'est pas reçue par son lecteur au sens où un message émis par une source est effectivement reçu par un destinataire. En d'autres termes, et de façon quelque peu paradoxale, le lecteur d'une étiquette assume plutôt le rôle de témoin contingent : lire une étiquette ne présuppose pas qu'elle ait été produite pour qui la lit (...) La présence du "producteur" de l'étiquette au moment où il la relie à son objet ne nous autorise même pas à le considérer comme un énonciateur
}

Outre le fait que les énoncés adhérents ne sont pas produits par des locuteurs humains en interaction, ils s'écartent du modèle dominant de la communication parce qu'ils sont indissociables de supports matériels. On est loin des corpus familiers aux analystes du discours où la dimension verbale est prépondérante et dont les enjeux sociaux sont immédiatement interprétables en termes de positionnement idéologique. En outre, comme le Web, mais pour des raisons très différentes, les énoncés adhérents échappent à la logique du texte conçu comme une totalité à l'aune de laquelle les autres données discursives sont évaluées. Certes, certains énoncés adhérents sont de courts textes, des suites de phrases soumises à des contraintes de cohésion et de cohérence, mais la plupart du temps ce sont des unités non-textuelles (groupes nominaux, phrases, suites de chiffres ou de lettres, logos...) qui sont dispersées sur un même support et qu'on ne peut pas unifier sous le foyer d'une conscience surplombante.

De manière plus générale, beaucoup d'analystes du discours sont mal à l'aise avec les agencements matériels associés aux énonciations. Quand ils étudient une allocution un meeting électoral, ils s'intéressent peu au décor, aux microphones ou aux écrans géants, mais concentrent l'essentiel de leur attention à l'argumentation. Sur ce point, l'analyse du discours aurait intérêt à mieux prendre en compte les problématiques des sciences humaines et sociales qui mettent l'accent sur les conditions techniques de l'énonciation. On peut songer en particulier aux nombreux travaux sur l'écriture à la suite de J. Goody (1977) et W. Ong (1982), au développement d'une "médiologie" (DEBRAY, 1991), à l'anthropologie des sciences qui confère un rôle central aux "non-humains" (LATOUR, 2007), aux modèles de cognition distribuée issus des sciences cognitives. Indépendamment de ces courants, certaines recherches à la croisée des sciences du langage et d'autres champs accordent toute leur place aux relations entre humains et artefacts, en particulier pour l'étude des rapports entre langage et travail (BOUTET; GARDIN; LACOSTE, 1995). De la même manière que le travail est de moins en moins une activité physique, que le langage s'intègre à tous ses aspects, les objets sont de moins en moins vierges de tout langage, et l'analyse du discours peut difficilement ne pas en prendre acte. 


\section{UN CHANGEMENT DE PARADIGME?}

J'ai évoqué divers ensembles de données qui sont peu ou pas du tout pris en compte par l'analyse du discours: les discours constituants, le Web, les locuteurs angéliques, les échanges avec les animaux, les OEuvres, les énoncés adhérents. Cette liste ne prétend pas être exhaustive. J'aurais pu par exemple y ajouter ce que j'appelle les aphorisations, les phrases sans texte (MAINGUENEAU, 2012), dont le dispositif d'énonciation s'écarte du modèle dominant de la communication : elles ne relèvent pas de la catégorie du genre de discours, leurs énonciateurs ne sont pas des locuteurs et ils n'ont pas de destinataires spécifiés.

Ce sont là des données disparates, dont le point commun est seulement d'être marginalisées par les approches usuelles des analystes du discours. Si je les ai ainsi mentionnées, c'est bien entendu parce que cette situation me paraît anormale, que l'analyse du discours devrait mieux intégrer les manifestations du discours dans toute leur diversité, quitte à mettre en question certains de ses présupposés. Mais c'est là une position qui peut être discutée. On peut en effet objecter que ces phénomènes sont intéressants mais qu'il n'y a pas de raison d'en confier l'étude à l'analyse du discours. L'étude du Web, par exemple, serait l'affaire des sciences de la communication et les énoncés adhérents, en fonction des types d'énoncés concernés, seraient de la compétence de la sociologie, de la sociolinguistique, de la sémiotique, de l'anthropologie, de la médiologie... Autrement dit, les études de discours n'auraient aucune raison de dévier fondamentalement de la ligne qu'elles suivent depuis qu'elles ont émergé, dans les années 1960. C'est une position défendable, à la condition que ceux qui la soutiennent disent en vertu de quels principes ils trouvent normal d'exclure telles ou telles données du champ de l'analyse du discours. Ce qui n'est pas si facile, car, comme je l'ai dit plus haut, dans leur grande majorité les analystes du discours ont jusqu'ici largement éludé ce type de questionnement.

Mais en ne mettant pas en question ses présupposé, en se repliant, comme on dit, sur sa zone de confort, l'analyse du discours se retrouve dans une situation d'inconfort épistémologique. Comme les données ainsi négligées par les études de discours représentent une masse considérable et en accroissement constant, le champ de analyse du discours apparaît de plus en plus restreint. Cette situation serait peu dommageable si les types d'activité discursive étaient bien disjoints ; le problème est que ces données ignorées ou marginalisées se mêlent aux corpus qu'affectionnent les analystes du discours. La réalité du discours, c'est une interpénétration profonde et généralisée des régimes de discours : nous ne cessons de combiner l'oral spontané, l'écrit traditionnel, l'écrit numérique, la navigation sur le web, les énoncés littéraires ou religieux, les énoncés adhérents...

Certains peuvent fort bien accepter que le champ du discours s'étende à de nouveaux corpus, tout en considérant que cela ne les concerne pas, que cela ne modifie pas leur 
manière d'étudier leurs propres corpus. En réalité, s'il y a ainsi extension du champ du discours, c'est la configuration dans son ensemble qui s'en trouve modifiée. Que se passe-t-il si le discours n'a plus de centre, qu'il est partagé entre plusieurs régimes? Pour prendre un exemple quelque peu caricatural, l'oralité a changé de statut quand on a inventé l'écriture : on ne pouvait plus l'appréhender de la même manière; devenue oralité " primaire ", elle s'est vue affecter, par sa simple confrontation avec l'écriture, de propriétés qui n'apparaissaient pas quand elle régnait sans partage. Le fait d'intégrer pleinement l'étude d'Internet, des énoncés adhérents ou des discours constituants au champ de l'analyse du discours fait que les corpus traditionnels ainsi que l'ensemble des outils analytiques apparaissent sous un nouveau jour et se voient conférer de nouvelles propriétés. L'enrichissement de l'univers du discours contraint à repenser un certain nombre de catégories et des procédures.

Face à une telle situation, on est tenté de dire que l'analyse du discours doit changer de paradigme, en faisant intervenir ici la célèbre notion (" paradigm shift ") issue de l'épistémologie anti-empiriste de Kuhn (1962). Le paradigme chez lui oscille entre une acception sociologique au sens large (un ensemble de valeurs, de croyances communes aux membres d'un groupe) et une acception heuristique (un ensemble de procédés de résolution des énigmes scientifiques). Son idée essentielle est qu'à une époque déterminée les chercheurs d'une même discipline disposent d'un cadre partagé, d'un ensemble de principes implicites qui définissent une "science normale ». Mais de temps en temps, quand l'ampleur des problèmes non résolus par le système existant devient trop grande, il se produit une crise qui amène à élaborer un nouveau paradigme. Cette représentation de l'histoire des sciences s'appuie implicitement sur la psychologie gestaltiste : d'une part les formes qui bloquent la perception des éléments divergents (la "science normale "), de l'autre les restructurations globales de la perception (les "révolutions scientifiques").

Mais appliquer cette problématique à l'analyse du discours ne va pas de soi. Kuhn étudie l'histoire de sciences telles que la physique où les chercheurs proposent des solutions concurrentes pour résoudre des problèmes qui se posent à tous. En revanche, en analyse du discours - comme d'ailleurs dans l'ensemble des sciences sociales - les chercheurs disposent de multiples ressources pour ne pas faire évolver le paradigme. Ils peuvent décider que les phénomènes qui n'entrent pas dans le cadre relèvent d'autres disciplines ou encore, s'ils relèvent de la même discipline, qu'ils ne sont pertinents que pour telle ou telle approche du discours. C'est en effet une des caractéristiques des sciences dites « molles " qu'il existe de multiples approches qui se présentent comme incommensurables, en d'autres termes qui définissent leur propre paradigme.

Dans ces conditions, il est très difficile d'imposer l'idée qu'il faudrait modifier en profondeur notre conception de la discursivité. L'intérêt d'un élargissement de l'analyse du discours ne s'impose que si l'on admet deux principes. Le premier est qu'il faut envisager les manifestations du discours dans toute leur diversité ; même si on travaille sur un secteur 
très limité de l'activité discursive, on doit aussi se préoccuper de la configuration d'ensemble dans laquelle il s'inscrit. Le second est que l'analyse du discours s'inscrit dans l'histoire, qu'elle est obligée de prendre en compte les changements qui se produisent dans les pratiques discursives et d'ajuster en conséquence ses concepts et ses méthodes. Certes, il ne saurait être question de constamment disqualifier les problématiques antérieures pour se tenir au plus près des changements ; mais il ne saurait être question non plus de s'arcbouter aux présupposés auxquels nous sommes accoutumés, en considérant que d'une certaine façon tout a déjà été dit dès l'origine par ceux qui ont contribué à fonder la discipline. Ce serait adopter une attitude proche de l'herméneutique religieuse, qui consiste à chercher, par une lecture appropriée de textes jugés fondateurs, les réponses à tous les problèmes. La bonne attitude se situe entre ces deux excès symétriques, mais comment définir ce point d'équilibre? C'est là une question qui est vouée à rester ouverte.

L'analyse du discours est apparue dans une certaine configuration historique. En organisant son champ autour des conversations et des genres institutionnels elle a pu se donner une relative homogénéité, mais ce geste a eu un coût : il a rejeté à la périphérie une multitude de données. Avec le temps cette situation devient difficilement tenable. L'analyse du discours devrait tirer toutes les conséquences du fait que l'univers du discours est hétérogène et dépourvu de centre si elle ne veut pas être réduite à un ensemble de "méthodes qualitatives" utilisée dans un domaine restreint des sciences sociales.

\section{RÉFÉRENCES}

ANGeRMUlLER, J.; MAINGUENEAU, D.; WODAK, R. (Ed.). The Discourse Studies Reader. Main Currents in Theory and Analysis. Amsterdam: John Benjamins, 2014.

AKRICH, M.; CALLON, M.; LATOUR, B. (Ed.). Sociologie de la traduction : textes fondateurs. Paris: Presses des Mines, 2006.

ARLUKE, A.; SANDERS, C. Regarding Animals. Animals, Culture, and Society. Philadelphia: Temple University Press, 1996.

BAZERMAN, C. Shaping Written Knowledge: the Genre and Activity of the Experimental Article in Science. lowa State University, 1988.

BOSREDON, B. Les titres de tableaux. Paris: PUF, 1997.

BOUTET, J.; GARDIN, B.; LACOSTE, M. Discours en situation de travail. Langages, n. 117, p. 12-31, 1995.

CHARAUDEAU, P. Une analyse sémiolinguistique du discours. Langages, n. 117, p. 96-111, 1995.

DEBRAY, R. Cours de médiologie générale. Paris: Gallimard, 1991.

DESCOLA P. Par-delà nature et culture. Paris: Gallimard, 2005.

FAIRCLOUGH, N. Analysing discourse. Textual analysis for social research. Routledge: London and New York, 2003. 
FOUCAULT. M. Qu'est-ce qu'un auteur ?. In: Dits et écrits /. 1954-1988. Paris: Gallimard, 1994, p 789-821.

GOODE, D. Playing with my dog Katie. West Lafayette (Indiana): Purdue University Press, 2007.

GUILLO, D. Des chiens et des humains. Paris: Le Pommier, 2009.

GOODY, J. The Domestication of the Savage Mind. Cambridge: Cambridge University Press, 1977.

HERITAGE, J. Conversational analysis and institutional talk. In: SANDERS, R.; FITCH, K. (Ed). Handbook of Language and Social Interadtion. Erlbaum: Mahwah, NJ, 2005. p 103-148.

HERITAGE, J.; CLAYMAN, S. E. Talk in Action: Interaction. Identities and Institutions. Boston: Wiley Blackwell, 2010.

HOUDART, S.; THIERY, O. (Ed.). Humains, non-humains: comment repeupler les sciences sociales ?Paris: La Découverte, 2011.

KUHN, T. S. The Structure of Scientific Revolutions. Chicago: University of Chicago Press, 1962.

LATOUR, B. I'Espoir de Pandore. Pour une version réaliste de l'activité scientifique. Paris: La Découverte, 2007.

MAINGUENEAU, D. Analysing self-constituting discourses. Discourse studies, v. 1, n. 2, p. 175-200, 1999.

MAINGUENEAU, D. Analyser les textes de communication. Paris: Dunod, 1998.

MAINGUENEAU, D. La Littérature pornographique. Paris: Armand Colin, 2008.

MAINGUENEAU, D. La difficile émergence d'une analyse du discours religieux. Langage et Société, n. 130, p. 513, 2009 .

MAINGUENEAU, D. Les phrases sans texte. Paris: A. Colin, 2012.

MAINGUENEAU, D. Genre de discours et Web : existe-t-il des genres Web ? In BARATS, C. (Ed.). Manuel d'analyse du Web en sciences humaines et sociales. Paris: A. Colin, 2013. p. 74-93.

MAINGUENEAU, D. The heterogeneity of discourse: expanding the field of discourse analysis. Palgrave Communications, n. 3, 2017. En ligne sur: http://www.palgrave-journals.com/articles/palcomms201758. Consulté le $1^{\circ}$ octobre 2020

MAINGUENEAU, D. Variações sobre o ethos. São Paulo: Parábola, 2020.

MONDÉMÉ, C. Comment parle-t-on aux animaux ? Formes et effets pragmatiques de l'adresse aux animaux de compagnie. Langage et Société, n. 163, p. 77-99, 2018.

MONDÉMÉ, C. La socialité interspécifique. Pour une analyse multimodale des interactions hommes-chiens. Limoges: Lambert-Lucas, 2019.

ONG, W. Orality and Literacy: The Technologizing of the Word. London \& New York: Routledge, 1982.

PATRIMOINE CULTUREL IMMATÉRIEL. Le chant de plein air des laboureurs. Dariolage, briolage... Recherches sur une tradition au Pays de la Châtaigneraie. Paris: L'Harmattan, 2012.

RASTIER, F. Arts et Sciences du texte. Paris: PUF, 2001.

SANDERS, C. Action Speak Louder than Words: Close Relationship Between Humans and Non-Human Animals. Symbolic Interaction, v. 26, n. 3, p. 405-426, 2003.

SWALES, J. M. Genre Analysis: English in Academic and Research Settings. Cambridge: Cambridge University Press, 1990. 